\title{
CONJUGACY EXPANSIVENESS IN FINITE GROUPS
}

\author{
ZOLTÁN HALASI, ATTILA MARÓTI, SAID SIDKI, AND MARCELO BEZERRA
}

\begin{abstract}
A finite group $G$ is called expansive if for every normal set $S$ and every conjugacy class $C$ of $G$ the normal set $S C$ consists of at least as many conjugacy classes of $G$ as $S$ does. This notion is motivated by a finiteness criterion. It is shown that a group is expansive if and only if it is a direct product of expansive simple or abelian groups. The groups $\operatorname{PSL}(2, q)$ and $\operatorname{Suz}(q)$ are expansive for every $q \geq 4$ and every $q=2^{2 n+1} \geq 8$ respectively. Many small simple groups including all sporadic simple groups are also expansive.
\end{abstract}

Dedicated to Geoffrey R. Robinson on the occasion of his 60th birthday.

\section{INTRODUCTION}

The topic of the present paper concerns an "expansive action" of a group which is defined using the set of conjugacy classes of the group. Motivated by a finiteness criterion [19, Section 3] the following general notion was suggested by the third author in his lecture at the conference Finite Groups and their Automorphisms in Istanbul, 2011.

For a non-empty set $X$ let $P$ be a partition of $X$ with finitely many parts. A subset of $X$ is called a $P$-set provided it is a union of parts of $P$. For an arbitrary subset $T$ of $X$ put $\|T\|_{P}=|\{S \in P \mid T \cap S \neq \emptyset\}|$.

Suppose a group $G$ acts on the set $X$ on the right. We say that the action of $G$ on $X$ is expansive with respect to the partition $P$ if $\|T\|_{P} \leq\|T g\|_{P}$ for every $g \in G$ and every $P$-set $T$. (This gives rise to a so-called special quadruple [19, Definition 3.1].) For example, the action of any finite group on any finite set is expansive with respect to any partition with equal parts. In particular, the right regular action of a finite group $G$ on itself is expansive with respect to the set of left cosets of any subgroup of $G$.

In this paper we consider the right regular action of $G$ on $X=G$ where $P$ is the set of conjugacy classes of $G$. Here a $P$-set $T$ is simply a normal subset of $G$ and $\|T g\|_{P}=\left\|T \cdot \mathcal{C}_{g}\right\|_{P}$ where $g \in G$ and $\mathcal{C}_{g}$ denotes the conjugacy class of $g$ in $G$. If

Date: January 24, 2012.

2000 Mathematics Subject Classification. 20E45, 20C15.

Key words and phrases. conjugacy class, finite group.

The research of the second author was supported by a Marie Curie International Reintegration Grant within the 7th European Community Framework Programme, by the János Bolyai Research Scholarship of the Hungarian Academy of Sciences, and by OTKA K84233. The third author acknowledges support from the Brazilian Scientific Agency CNPq. 
a finite group $G$ is such that this action of $G$ on $X$ is expansive with respect to $P$ then we simply say that $G$ is expansive.

To motivate this latter notion we state a special case of [19, Theorem 3.5] (here there is a misprint: $m$ and $n$ should be interchanged). Let $G$ be a non-trivial expansive group. Put $n=|G|$ and $k=k(G)$ where $k(G)$ denotes the number of conjugacy classes of $G$. Let $K$ be an arbitrary group of order $k$ and let $\gamma$ be a (surjective) map from $G$ to $K$ so that the preimage of any element of $K$ is a conjugacy class of $G$ and that the preimage of 1 is $\{1\}$. Moreover let $\delta$ and $\epsilon$ be any functions from $G$ to $K$ and $G$ to $G$ respectively. Then the finitely presented group $\left\langle G, K \mid g \gamma(g) \epsilon(g)^{-1} \delta(g)^{-1}=1, \quad \forall g \in G\right\rangle$ is finite of order at most $(n-1) e^{n}$ where $e$ is the base of the natural logarithm.

The subject of products of conjugacy classes in finite groups has received wide interest. See the book [1] by Arad and Herzog or the papers citing it.

The product of two conjugacy classes in a non-abelian finite simple group $G$ tend in general to decompose into a large number of conjugacy classes. In particular, a conjecture of J. G. Thompson saying that there exists a conjugacy class $C$ in $G$ so that $G=C C$ has been the focus of much research on this topic. For example, Guralnick and Malle [10, Theorem 1.4] recently proved that there exist conjugacy classes $C_{1}$ and $C_{2}$ in $G$ with $G=C_{1} C_{2} \cup\{1\}$.

A preliminary study indicated that the expansiveness of a finite group implies strong restrictions on its structure. The main results of this paper confirm this view.

We say that a finite group $G$ is normal expansive if for every normal subgroup $N$ of $G$ and every conjugacy class $C$ of $G$ the normal set $N C$ is the union of at least as many conjugacy classes of $G$ as $N$ is. Clearly, an expansive group is also normal expansive. As above, for a finite group $H$ we denote the number of conjugacy classes of $H$ by $k(H)$.

Theorem 1.1. For a finite group $G$ the following conditions are equivalent.

(i) $G$ is normal expansive.

(ii) $G$ is a direct product of simple or abelian groups.

(iii) $k(G)=k(N) k(G / N)$ for every normal subgroup $N$ of $G$.

(iv) $C_{G}(x \bmod N)=C_{G}(x) N$ for every $x \in G$ and every normal subgroup $N$ of $G$.

(v) For every normal subgroup $N$ of $G$ and any elements $x, y$ of $G$ so that $[x, y] \in N$, any irreducible character of $N$ extends to an irreducible character of $N\langle x, y\rangle$.

(vi) For every irreducible character $\chi$ of any normal subgroup $N$ of $G$ the number of irreducible characters of $G$ lying above $\chi$ is $\left|G: \operatorname{Stab}_{G}(\chi)\right| \cdot k(G / N)$.

(vii) Every irreducible character of any normal subgroup of $G$ can be extended to $G$.

(viii) Every coset of any fixed normal subgroup of $G$ meets the same number of conjugacy classes of $G$.

Our proof of the implication (i) $\Rightarrow$ (ii) of Theorem 1.1 depends on the Classification of Finite Simple Groups. 
Using Hall's Marriage Theorem and Theorem 1.1 the following is proven.

Theorem 1.2. A group is expansive if and only if it is a direct product of expansive simple or abelian groups.

This theorem tells us that the next step is to determine which simple groups are expansive. Next we prove

Theorem 1.3. Let $G$ be $\operatorname{PSL}(2, q)$ for some $q \geq 4$, or $\operatorname{Suz}(q)$ for some $q=2^{2 n+1}$ with $n \geq 1$, or one of the 26 sporadic simple groups. Then $G$ is expansive.

Given a character table of a finite group in Gap [8], the structural constants of the group can be determined. With these, in conjunction with an implementation of the Hungarian Algorithm [15] we were able to check that all the 138 simple groups in the Gap Character Table Library (including all the sporadic simple groups) are indeed expansive. (The result of these computer calculations can be found at [11].) This lead us to state the following conjecture.

Conjecture 1.4. Every finite simple group is expansive.

To support Conjecture 1.4 we mention a result of Gow [9, Theorem 2] saying that in any finite simple group of Lie type the product of any two regular semisimple conjugacy classes contains all semisimple conjugacy classes of the group.

Finally, in the last section, we prove a technical result on what can be said if the number of non-zero entries in a given column of the character table of the group is known.

\section{Reduction to Direct products}

In this section we prove Theorem 1.1.

First we show that (i) implies (ii).

Lemma 2.1. Let $N$ be a normal subgroup of a normal expansive group $G$. Then $G / N$ acts trivially on the set of conjugacy classes of $N$. In particular $N$ is normal expansive. Every subnormal subgroup of a normal expansive group is normal and normal expansive.

Proof. The last two statements are consequences of the first. Let $N$ be a normal subgroup of a normal expansive group $G$. Put $H=G / N$ and let $h$ be an element of $H$. By [14, Page 460] (which is also a consequence of any of the following: [13, Proposition 9.4], [3, Theorem 1], and [6, Lemma $2.2(2)]$ ), the number of $N$-conjugacy classes contained in the coset $h$ is equal to $k_{h}(N)$, the number of $h$ invariant conjugacy classes of $N$. In particular, the number of $G$-conjugacy class representatives contained in $h$ is at most $k_{h}(N)$. By the Orbit Counting Lemma, the number $n(G, N)$ of $G$-conjugacy classes in $N$ is $(1 /|H|) \sum_{y \in H} k_{y}(N)$. If $n(G, N)>$ $k_{h}(N)$ for some $h \in H$ then $G$ cannot be normal expansive just by considering the normal set $N x^{G}$ where $x$ is so that $h=N x$. This means that $k_{y}(N)$ must be the same for all $y \in H$. For $y=1$ this is $k(N)$, the number of conjugacy classes of $N$. We conclude that $H$ acts trivially on the set of conjugacy classes of $N$. 
Lemma 2.2. A solvable normal expansive group is abelian.

Proof. Let $G$ be a minimal counterexample to the statement of the theorem. Let $N$ be a proper normal subgroup of $G$ with $G / N$ cyclic. Then $N$ is abelian by the minimality of $G$ and the normal expansiveness of $N$. Again by Lemma 2.1, $G / N$ acts trivially on $N$. Hence $N \leq Z(G)$. But then $G$ is abelian, a contradiction.

Lemma 2.3. If a non-trivial central element of a finite group $G$ is a commutator, then $G$ cannot be normal expansive.

Proof. Let $z=[x, y]$ be a non-trivial central element in $G$ where $x$ and $y$ are elements of $G$. Then $\{1, z\} x=\left\{x, x^{y}\right\}$ which means that $\{1, z\} x^{G}=x^{G}$. From this it follows that the normal set $\langle z\rangle x^{G}$ is the union of less than $o(z)$ conjugacy classes of $G$. Hence $G$ cannot be normal expansive.

Lemma 2.4. A normal expansive quasisimple group is simple.

Proof. Let $G$ be a quasisimple group with $Z(G) \neq 1$. By Lemma 2.3, it is sufficient to see that some non-trivial element of $Z(G)$ is a commutator. First note that a central element $z$ in a finite group $H$ is a commutator in $H$ if and only if there is a conjugacy class $C$ of $H$ such that $C z=C$. Now apply [2, Theorem 1] to see that there is always a central element of prime order in $G$ which is a commutator in $G$ except possibly when $G / Z(G) \cong \operatorname{PSL}(3,4)$ and $Z(G)$ is non-cyclic. So suppose that $G$ is such an exceptional group. Let $N$ be a central subgroup in $G$ such that $Z(G) / N$ is a non-trivial cyclic group. Then $G / N$ is quasisimple with center $Z(G) / N$ so, again by [2, Theorem 1], there exists a non-trivial element $z N$ (of prime order) such that $z N=[x N, y N]$ for some elements $x$ and $y$ in $G$. In particular, $[x, y] \in z N$, so $[x, y]$ is a non-trivial central element in $G$.

Let $F(G)$ denote the Fitting subgroup of a finite group $G$. This is the largest nilpotent normal subgroup of $G$. Let $L(G)$ be the layer of a finite group $G$. This is the central product of all subnormal quasisimple groups of $G$ (if they exist). The group $F^{*}(G)=F(G) L(G)$ is called the generalized Fitting subgroup of $G$. This group has the property that $C_{G}\left(F^{*}(G)\right) \leq F^{*}(G)$.

Lemma 2.5. If $G$ is normal expansive then $F(G)=Z(G)$ and $L(G)$ is trivial or is a direct product of non-abelian simple groups.

Proof. Let $G$ be normal expansive. Then the nilpotent normal subgroup $F(G) \geq$ $Z(G)$ is normal expansive by Lemma 2.1. By Lemma 2.2, $F(G)$ is abelian. Again by Lemma $2.1, G / F(G)$ acts trivially on $F(G)$. Hence $F(G) \leq Z(G)$. We conclude that $F(G)=Z(G)$. Suppose now that $L(G) \neq 1$. Then every subnormal quasisimple group in $G$ is simple, by Lemma 2.1 and Lemma 2.4. Thus $L(G)$ is the direct product of these simple groups.

The following lemma finishes the proof of (i) implies (ii).

Lemma 2.6. A normal expansive group is a direct product of simple or abelian groups. 
Proof. Let $G$ be a normal expansive group. If $G=F^{*}(G)$ then there is nothing to prove by Lemma 2.5. If $L(G)=1$ then $F^{*}(G)=F(G)=Z(G)$ and so $G$ is abelian. Hence assume that $L(G) \neq 1$. Let $x \in G \backslash F^{*}(G)$. If $x$ permutes some of the simple factors of $L(G)$ then $x$ cannot act trivially on the set of conjugacy classes of $F^{*}(G)$ violating Lemma 2.1. Hence $x$ must act as an outer automorphism on some of the simple factors of $L(G)$ (since $C_{G}(L(G)) \leq F^{*}(G)$ ). But then, by [5, Theorem $\mathrm{C}], x$ will fuse some of the conjugacy classes of that simple factor together. This contradicts Lemma 2.1. Thus such an $x$ cannot exist and the proof is complete.

Now we complete the proof of Theorem 1.1.

The implication (ii) $\Rightarrow$ (iii) is trivial. The equivalence (iii) $\Leftrightarrow$ (iv) is proved in $[7$, Section 1] and the equivalence (iii) $\Leftrightarrow(\mathrm{v})$ is shown in [7, Section 3]. The equivalence (iii) $\Leftrightarrow$ (vi) follows from the argument in [18, Pages 97, 98]. The implication (ii) $\Rightarrow$ (vii) $\Rightarrow$ (v) is clear. The equivalence (iii) $\Leftrightarrow$ (viii) can be established from the proof of [17, Lemma 1]. Finally, it is clear that (viii) implies (i).

\section{REDUCtion to SIMPLE GROUPS}

In this section we prove Theorem 1.2.

We need a definition. Let $C$ be a conjugacy class of a finite group $G$. We say that $G$ is expansive for $C$ if for any normal set $S$ of $G$ the normal set $S C$ is a union of at least as many conjugacy classes of $G$ as $S$ is. (Clearly, $G$ is expansive if and only if $G$ is expansive for all of its conjugacy classes.)

Lemma 3.1. A finite group $G$ is expansive for a conjugacy class $C$ if and only if there exists a permutation $\pi$ of the set of conjugacy classes of $G$ such that $\pi(D) \subseteq$ $D C$ for all conjugacy classes $D$ of $G$.

Proof. Let $\mathcal{D}$ be the set of conjugacy classes of $G$. For $C \in \mathcal{D}$ we define a bipartite graph $\Gamma_{C}$ as follows. Let $X=\{(D, 1): D \in \mathcal{D}\}$ and $Y=\{(D, 2): D \in \mathcal{D}\}$. Let $X \cup Y$ be the set of vertices of $\Gamma_{C}$. Let there be an edge between vertices $\left(D_{1}, 1\right)$ and $\left(D_{2}, 2\right)$ if and only if $D_{2} \subseteq D_{1} C$. Then $G$ is expansive for $C$ if and only if the Hall Condition holds for $\Gamma_{C}$. Now Hall's Marriage Theorem [12] guarantees a perfect matching in $\Gamma_{C}$ which in turn gives a suitable permutation $\pi$ on $\mathcal{D}$.

Proposition 3.2. A direct product of finite groups is expansive if and only if every direct factor is expansive.

Proof. Clearly it is enough to prove the proposition for two direct factors $K$ and $L$.

Suppose that any of $K$ and $L$, say $K$, is non-expansive. Then there exists a normal set $S$ in $K$ and a conjugacy class $C$ in $K$ such that the number of conjugacy classes contained in $S C$ is less than the number of conjugacy classes contained in $S$. Then the normal set $S \times\{1\}$ in $G=K \times L$ and the conjugacy class $C \times\{1\}$ in $G$ fail the condition of expansiveness for $G$.

Suppose now that both $K$ and $L$ are expansive. Let $C=C_{K} \times C_{L}$ be an arbitrary conjugacy class in $G$ where $C_{K}$ is a conjugacy class of $K$ and $C_{L}$ is a conjugacy 
class of $L$. Let $C_{1}, \ldots, C_{n}$ be the conjugacy classes of $K$ and let $C_{n+1}, \ldots, C_{n+r}$ be the conjugacy classes of $L$. Let $\pi$ be a permutation of $\{1, \ldots, n\}$ such that $C_{i} C_{K} \supseteq C_{\pi(i)}$ for all $i$ with $1 \leq i \leq n$. Such a $\pi$ exists by Lemma 3.1. Let $\sigma$ be a permutation of $\{n+1, \ldots, n+r\}$ such that $C_{j} C_{L} \supseteq C_{\sigma(j)}$ for all $j$ with $n+1 \leq j \leq n+r$. Such a $\sigma$ exists again by Lemma 3.1. Then there is a permutation $\rho$ of the $n r$ conjugacy classes of $G$ such that

$$
\left(C_{i} \times C_{j}\right) C=C_{i} C_{K} \times C_{j} C_{L} \supseteq C_{\pi(i)} \times C_{\sigma(j)}=\rho\left(C_{i} \times C_{j}\right)
$$

for all $i$ and $j$ with $1 \leq i \leq n$ and $n+1 \leq i \leq n+r$. Hence $G$ is expansive by Lemma 3.1.

Now Theorem 1.2 follows from the equivalence (i) $\Leftrightarrow$ (ii) of Theorem 1.1 and from Proposition 3.2 .

\section{The Proof of TheOrem 1.3}

In this section we prove Theorem 1.3. Note that by the paragraph after the statement of Theorem 1.3, we only need to deal with the groups $\operatorname{PSL}(2, q)$ for $q \geq 4$ and $\operatorname{Suz}(\mathrm{q})$ for $q=2^{2 n+1} \geq 8$.

Proposition 4.1. The group $\operatorname{PSL}(2, q)$ is expansive for every $q \geq 4$.

Proof. To prove that $\operatorname{PSL}(2, q)$ is expansive for $q \geq 4$ we shall use a theorem of Macbeath [16, Theorem 1], which says that for any prime power $q$ and any triples $(\alpha, \beta, \gamma) \in \mathbb{F}_{q}$ there are matrices $A, B, C \in \mathrm{SL}(2, q)$ such that $(\operatorname{Tr} A, \operatorname{Tr} B, \operatorname{Tr} C)=$ $(\alpha, \beta, \gamma)$ and $A B C=1$.

First let us assume that the characteristic of $\mathbb{F}_{q}$ is 2 . Then $\operatorname{PSL}(2, q)$ consists of $q-1$ semisimple conjugacy classes and two unipotent conjugacy classes. Furthermore, the trace function provides a one-to-one correspondence between the semisimple classes and the non-zero field elements. Let $\{1\}=C_{1}, C_{2}, \ldots, C_{q+1}$ be the list of conjugacy classes of $G$ with $C_{2}$ being the non-trivial unipotent class. Let $R$ be any normal set with $1<\|R\|_{P}<q+1=k(\operatorname{PSL}(2, q))$ and let $C$ be any non-trivial class. By the theorem of Macbeath, for $3 \leq r, s \leq q+1$ each of the products $C_{r} C_{s}, C_{r}\left(C_{1} \cup C_{2}\right),\left(C_{1} \cup C_{2}\right) C_{2}$ contains at least $q$ conjugacy classes, and $C_{r} C_{2}$ contains any semisimple class except (possibly) $C_{r}$ and it also contains $C_{2}$. It follows that $\|R C\|_{P} \geq q \geq\|R\|_{P}$ unless $C=C_{2}$ and $R$ is the union of a unipotent and a semisimple class. But in this case $\|R\|_{P}=2$, while $\|R C\|_{P} \geq q-1 \geq 3$ since $q \geq 4$.

In the following let us assume that the characteristic of $\mathbb{F}_{q}$ is odd. We checked that $\operatorname{PSL}(2, q)$ is expansive for $q=5,7,9$ by using Gap [8], so we will assume that $q \geq 11$. Now, the conjugacy classes of $\mathrm{SL}(2, q)$ are characterized as follows. For any $\alpha \in \mathbb{F}_{q} \backslash\{ \pm 2\}$ the set $C_{\alpha}=\{A \in \mathrm{SL}(2, q) \mid \operatorname{Tr} A=\alpha\}$ is a unique conjugacy class, while for $\alpha \in\{ \pm 2\}$ it is the union of 3 classes, whose two non-central classes are conjugate in $G L(2, q)$. For example, for $\alpha=2$, the two non-trivial classes are represented by the matrices

$$
c=\left(\begin{array}{ll}
1 & 1 \\
0 & 1
\end{array}\right), \quad d=\left(\begin{array}{ll}
1 & \nu \\
0 & 1
\end{array}\right), \text { where }\langle\nu\rangle=\mathbb{F}_{q}^{\times} .
$$


As a consequence, in $\operatorname{PSL}(2, q)$ there are three unipotent conjugacy classes $C_{1}=$ $\{1\}, C_{c}, C_{d}$, and $\frac{1}{2}(q-1)$ non-trivial semisimple classes $\left\{C_{s} \mid s \in S\right\}$ characterized by the set $S=\left(\mathbb{F}_{q} \backslash\{ \pm 2\}\right) / \sim$, where $\sim$ is the equivalence relation defined by $\alpha \sim \beta \Longleftrightarrow \alpha^{2}=\beta^{2}$. Furthermore, there is a $\varphi$ outer automorphism of $\operatorname{PSL}(2, q)$ such that $\varphi\left(C_{c}\right)=C_{d}, \varphi\left(C_{d}\right)=C_{c}$ and $\varphi(C)=C$ for any other conjugacy class. (Such a $\varphi$ can be represented by conjugation with the diagonal matrix $\operatorname{diag}(1, \nu) \in$ $G L(2, q))$. Let $C$ be a non-trivial conjugacy class and let $R$ be any normal set. We show that $\|R\|_{P} \leq\|R C\|_{P}$ holds.

First, let $C \in\left\{C_{s} \mid s \in S\right\}$ be a semisimple class. Let us assume that there is a non-trivial class $C_{r} \subseteq R$. By using the above mentioned theorem of Macbeath, and also the existence of the automorphism $\varphi$, it follows that $C_{r} C \subseteq R C$ contains any semisimple class $C_{s}$ with $s \in S$ except possibly $C$ (for which $C \subseteq C_{r} C$ holds if $C_{r}$ is semisimple) and at least one unipotent class. So, $\|R\|_{P} \leq\|R C\|_{P}$ unless $\|R\|_{P} \geq k(P S L(2, q))-2$. Let us assume that this is the case. Using that $q \geq 9$, we get that there are at least 4 elements in $\left\{C_{s} \mid s \in S\right\}$ so there is a non-trivial semisimple conjugacy class $C_{r} \subseteq R$ such that $C_{r} \neq C^{-1}=C$, so $C_{1} \nsubseteq \subseteq C_{r} C$. As $C_{r} C$ contains a unipotent class, it contains either $C_{c}$ or $C_{d}$. Using $\varphi$ we get $C_{r} C$ contains both $C_{c}$ and $C_{d}$. Since $C_{r} C$ also contains every non-trivial semisimple class we get that $\|R C\|_{P} \geq k(\operatorname{PSL}(2, q))-1$ holds, and we are done.

Now, let $C \in\left\{C_{c}, C_{d}\right\}$. First, we prove that if $R \subseteq C_{1} \cup C_{c} \cup C_{d}$ is a normal set, then $R C$ contains at least as many unipotent classes as $R$ does. To see this, let $P \subseteq \operatorname{PSL}(2, q)$ be the homomorphic image of the subgroup of unipotent upper triangular matrices in $\operatorname{SL}(2, q)$ through the natural homomorphism $S L(2, q) \rightarrow$ $\operatorname{PSL}(2, q)$. Then $P=P_{1} \cup P_{c} \cup P_{d}$ is a partition of $P$ to non-empty subsets where $P_{t}=P \cap C_{t}$ for $t \in\{1, c, d\}$. Now, $|(R \cap P) \cdot(C \cap P)| \geq|R \cap P|$, which proves that $R C \cap P$ contains at least as many members of $\left\{P_{1}, P_{c}, P_{d}\right\}$ as $R \cap P$, which proves the statement. As a result, we also get $\|R\|_{P} \leq\|R C\|_{P}$ if $R$ does not contain any non-trivial semisimple class.

Now, let us assume that $R$ contains a non-trivial semisimple class $C_{r} \subseteq\left\{C_{s} \mid s \in\right.$ $S$ \}. Then $C_{r}\left(C_{1} \cup C_{c} \cup C_{d}\right)$ contains any non-trivial semisimple class, so $C_{r} C$ contains any class in $\left\{C_{s} \mid s \in S \backslash\{r\}\right\}$. So, we are done unless $\|R\|_{P} \geq k(\operatorname{PSL}(2, q))-3$. Let us assume that this is the case. As $q \geq 11$, there are at least five non-trivial semisimple classes in $\operatorname{PSL}(2, q)$. It follows that $R$ contains at least two such classes and $R C$ contains all the non-trivial semisimple classes. In view of our previous statement on the number of unipotent classes in $R$ and in $R C$, the result $\|R\|_{P} \leq\|R C\|_{P}$ follows.

Now we turn to the Suzuki groups.

Proposition 4.2. The group $\operatorname{Suz}(q)$ is expansive for every $q=2^{2 n+1}$ with $n \geq 1$.

Proof. The (simple) Suzuki group $\operatorname{Suz}(q)$ has $q+3$ conjugacy classes; 4 unipotent classes and $q$ semisimple classes. The four unipotent classes are the trivial class $C_{1}$, the class of involutions $C_{2}$, and two classes of elements of orders 4 which we denote by $C_{3}$ and $C_{4}$. Let $R \neq C_{1}$ be an arbitrary normal set and let $C \neq C_{1}$ be a conjugacy class of $\operatorname{Suz}(q)$. We must show that $\|R\|_{P} \leq\|R C\|_{P}$. 
To prove this we rely on a result found in the proof of Theorem 4.1 in [1, Page $239]$ which states that the product of any two non-trivial conjugacy classes in $G=$ $\operatorname{Suz}(q)$ contains all non-trivial elements of $G$ except in the following cases: $C_{2}^{2}=$ $G \backslash\left(C_{3} \cup C_{4}\right)$ and $C_{2} C_{3}=C_{3} C_{2}=C_{2} C_{4}=C_{4} C_{2}=G \backslash\left(C_{1} \cup C_{2}\right)$. This means that we certainly have $q+1 \leq\|R C\|_{P}$ in all cases. Thus we may assume that $\|R\|_{P}=q+2$. But then $R$ contains a semisimple conjugacy class $D$ and by the result mentioned above $D C$ contains all non-trivial elements of $G$.

\section{An ADDitional RESUlt}

For the last result of this paper we need a definition. As before, let $k(G)$ be the number of conjugacy classes of a finite group $G$. For an integer $n$ with $1 \leq n \leq k(G)$ and a conjugacy class $C$ of $G$ we say that $G$ is $n$-expansive for $C$ if there exists a set $\mathcal{C}$ consisting of $n$ distinct conjugacy classes of $G$ such that whenever $S$ is a normal set of $G$ which is a union of some elements of $\mathcal{C}$ then the normal set $S C$ is the union of at least as many conjugacy classes of $G$ as $S$ is. Every group is 1-expansive for every conjugacy class of the group. If $G$ is $k(G)$-expansive for a conjugacy class $C$ of $G$ then $G$ is expansive for $C$.

Proposition 5.1. Let $C$ be a conjugacy class of a finite group G. Suppose that there are $n$ non-zero entries in the column associated to $C$ in the character table of $G$. Then $G$ is n-expansive for $C$.

Proof. Let $C_{1}, \ldots, C_{s}$ be the conjugacy classes of $G$. Let $C=C_{j}$ for some $j$. For every $i$ in $\{1, \ldots, s\}$ let $K_{i}=\sum_{x \in C_{i}} x$ and put $K_{i} K_{j}=\sum_{k=1}^{s} c_{i j k} K_{k}$ where the $c_{i j k}$ are non-negative integers called the structure constants. As on [4, Page 235] let $V_{j}$ be the matrix $\left[c_{i j k}\right]_{1 \leq i, k \leq s}$. Let $n$ be as in the statement of the proposition. By Lemma 3.1, $G$ is $n$-expansive for $C$ if and only if there exist $n$ rows and $n$ columns in $V_{j}$ so that there exist $n$ non-zero entries in the matrix $V_{j}$ with exactly one entry in each row of the chosen $n$ rows and exactly one entry in each of the chosen $n$ columns. This clearly holds if some minor of $V_{j}$ of order $n$ is non-zero, in other words if the rank of $V_{j}$ is at least $n$. Hence it is sufficient to show that the rank of the matrix $V_{j}$ is at least $n$.

Let the complex irreducible characters of $G$ be $\chi_{1}, \ldots, \chi_{s}$. For every integer $i$ and $r$ between 1 and $s$ let $\omega_{i}^{(r)}=\left|C_{i}\right| \chi_{r}\left(x_{i}\right) / \chi_{r}(1)$ where $x_{i} \in C_{i}$. By [4, Page 235], the eigenvalues of the matrix $V_{j}$ are $\omega_{j}^{(1)}, \ldots, \omega_{j}^{(s)}$, and by [4, Page 237], the associated eigenvectors are $v_{1}, \ldots, v_{s}$ where $v_{r}=\left[\omega_{1}^{(r)}, \ldots, \omega_{s}^{(r)}\right]^{T}$ for every $r$ with $1 \leq r \leq s$. Since

$$
\left[v_{1}, \ldots, v_{s}\right]^{T}=\operatorname{diag}\left(\frac{1}{\chi_{1}(1)}, \ldots, \frac{1}{\chi_{s}(1)}\right) \cdot M \cdot \operatorname{diag}\left(\left|C_{1}\right|, \ldots,\left|C_{s}\right|\right)
$$

where $M$ is the character table of $G$ and since the determinant of $M$ is non-zero, the determinant of $\left[v_{1}, \ldots, v_{s}\right]^{T}$ is also non-zero. Thus the vectors $v_{1}, \ldots, v_{s}$ are linearly independent. Hence, in this basis, the matrix $V_{j}$ becomes $\operatorname{diag}\left(\omega_{j}^{(1)}, \ldots, \omega_{j}^{(s)}\right)$ which clearly has rank $n$. This finishes the proof of the proposition. 
Acknowledgment. We thank the anonymous referee for pointing out the reference [16] which lead us to the present simpler proof of the expansiveness of $\operatorname{PSL}(2, q)$ for $q \geq 4$.

\section{REFERENCES}

[1] Arad, Z.; Herzog, M. Products of conjugacy classes in groups. Lecture Notes in Mathematics, 1112. Springer-Verlag, Berlin, 1985.

[2] Blau, H. I. A fixed-point theorem for central elements in quasisimple groups. Proc. Amer. Math. Soc. 122 (1994), no. 1, 79-84.

[3] Britnell, J. R.; Wildon, M. Commuting conjugacy classes: an application of Hall's marriage theorem to group theory. J. Group Theory 12 (2009), no. 6, 795-802.

[4] Curtis, C. W.; Reiner, I. Representation theory of finite groups and associative algebras. Reprint of the 1962 original. AMS Chelsea Publishing, Providence, RI, 2006.

[5] Feit, W.; Seitz, G. M. On finite rational groups and related topics. Illinois J. Math. 33 (1989), no. $1,103-131$

[6] Fulman, J.; Guralnick, R. M. Bounds on the number and sizes of conjugacy classes in finite Chevalley groups with applications to derangements. arXiv:0902.2238

[7] Gallagher, P. X. The number of conjugacy classes in a finite group. Math. Z. 118 (1970) 175-179.

[8] The GAP Group, GAP - Groups, Algorithms, and Programming, Version 4.4.10; 2007. (http://www.gap-system.org)

[9] Gow, R. Commutators in finite simple groups of Lie type. Bull. London Math. Soc. 32 (2000), no. 3, 311-315.

[10] Guralnick, R. M.; Malle, G. Products of conjugacy classes and fixed point spaces. J. Amer. Math. Soc. 25 (2012), 77-121.

[11] http://www.renyi.hu/ zhalasi/expansive

[12] Hall, P. On representatives of subsets. J. London Math. Soc. 10 (1) (1935) 26-30.

[13] Isaacs, I. M. Characters of $\pi$-separable groups. J. Algebra 86 (1984), no. 1, 98-128.

[14] Keller, T. M. Fixed conjugacy classes of normal subgroups and the k(GV)-problem. J. Algebra 305 (2006), no. 1, 457-486.

[15] Kuhn, H. W. The Hungarian method for the assignment problem. Naval Res. Logist. Quart. 2 (1955) 83-97.

[16] Macbeath, A. M. Generators of the linear fractional groups. 1969 Number Theory (Proc. Sympos. Pure Math., Vol. XII, Houston, Tex., 1967) pp. 14-32 Amer. Math. Soc., Providence, R.I.

[17] Nagao, H. On a conjecture of Brauer for p-solvable groups. J. Math. Osaka City Univ. 13 (1962) 35-38.

[18] Robinson, G. R. Representation theory notes, unpublished.

[19] Sidki, S. On weak permutability between groups. J. Algebra 63 (1980) 186-225.

Department of Algebra and Number Theory, Institute of Mathematics, University of Debrecen, 4010, Debrecen, Pf. 12, Hungary

E-mail address: zhalasi@renyi.hu

mTA Alfréd Rényi Institute of Mathematics, Reáltanoda utca 13-15, H-1053, BuDAPEST, Hungary

E-mail address: maroti@renyi.hu

Departamento de Matemática, Universidade de Brasília, Brasília, DF 70910-900, Brazil

E-mail address: ssidki@gmail.com

Departamento de Matemática, Universidade de Brasília, Brasília, DF 70910-900, Brazil

E-mail address: bezerr4@gmail.com 